\title{
Corynebacterium ulcerans 0102 carries the gene encoding diphtheria toxin on a prophage different from the C. diphtheriae NCTC 13129 prophage
}

Tsuyoshi Sekizuka', Akihiko Yamamoto², Takako Komiya², Tsuyoshi Kenri², Fumihiko Takeuchi ${ }^{1}$, Keigo Shibayama², Motohide Takahashi ${ }^{2,3}$, Makoto Kuroda ${ }^{1}$ and Masaaki Iwaki ${ }^{2^{*}}$

\begin{abstract}
Background: Corynebacterium ulcerans can cause a diphtheria-like illness, especially when the bacterium is lysogenized with a tox gene-carrying bacteriophage that produces diphtheria toxin. Acquisition of toxigenicity upon phage lysogenization is a common feature of $C$. ulcerans and C. diphtheriae. However, because of a lack of $C$. ulcerans genome information, a detailed comparison of prophages has not been possible between these two clinically important and closely related bacterial species.

Results: We determined the whole genome sequence of the toxigenic C. ulcerans 0102 isolated in Japan. The genomic sequence showed a striking similarity with that of Corynebacterium pseudotuberculosis and, to a lesser extent, with that of $C$. diphtheriae. The 0102 genome contained three distinct prophages. One of these,

DCULC0102-I, was a tox-positive prophage containing genes in the same structural order as for tox-positive C. diphtheriae prophages. However, the primary structures of the individual genes involved in the phage machinery showed little homology between the two counterparts.
\end{abstract}

Conclusion: Taken together, these results suggest that the tox-positive prophage in this strain of C. ulcerans has a distinct origin from that of C. diphtheriae NCTC 13129.

Keywords: Bacteriophage, Toxin gene, Horizontal gene transfer, Diphtheria, Zoonosis

\section{Background}

A diphtheria-like infectious disease caused by Corynebacterium ulcerans is increasing in clinical importance in developed countries and is now regarded as "diphtheria" in Europe [1,2]. Infection with C. ulcerans occurs in a wide range of hosts, including cats, dogs, pigs, cows, and whales [3-9]. The first clearly documented case of zoonotic transmission involved a dog, as reported by Lartigue et al. [5]. This is in contrast to the causative agent of classical diphtheria, C. diphtheriae, whose host species is thought to be limited to humans [10]. Nevertheless, the two species share a common feature: upon

\footnotetext{
* Correspondence: miwaki@nih.go.jp

${ }^{2}$ Department of Bacteriology II, National Institute of Infectious Diseases, 4-7-1 Gakuen, Musashimurayama-shi, Tokyo 208-0011, Japan

Full list of author information is available at the end of the article
}

lysogenization of tox-encoding bacteriophages, they become toxigenic and are able to produce the potent diphtheria toxin $[1,10]$. This toxin is known to contribute to disease progression, occasionally leading to death. It is encoded by a single gene designated tox, situated inside prophages lysogenized in the bacterial genome of $C$. diphtheriae [11]. The prophages are capable of induction, by ultraviolet light or DNA-damaging agents such as mitomycin $C$, and yield $\beta-, \delta-, \omega$ - and other functional bacteriophage particles [12]. Some types of bacteriophages can infect both $C$. diphtheriae and C. ulcerans [13-16]. Furthermore, the C. ulcerans tox gene is also encoded in a genome region surrounded by phage attachment (att) sites conserved between the two species $[7,16]$. The nucleotide sequences of C. ulcerans tox genes were published by Sing et al. They showed some 


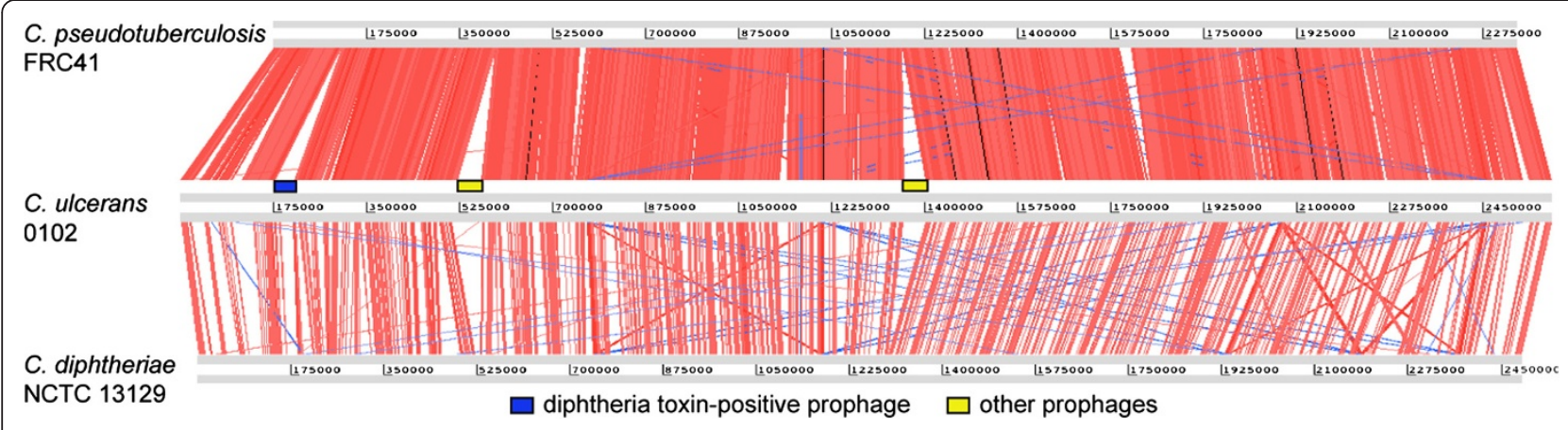

Figure 1 Schematic genome comparison. C. ulcerans 0102 (middle) with C. pseudotuberculosis FRC41 (top) and C. diphtheriae NCTC 13129 (bottom) using a BLASTN homology search visualized by the ACT program. The red and blue bars between chromosomal DNA sequences represent individual nucleotide matches in the forward and reverse directions, respectively. BLASTN match scores less than 200 are not shown. A blue box and two yellow boxes represent a tox-positive prophage and other prophages on the chromosome of C. ulcerans 0102 , respectively.

diversity in the genetic sequence among C. ulcerans strains, in contrast to the highly conserved $C$. diphtheriae tox gene $[17,18]$.

In 2003, the nucleotide sequence of the whole genome of C. diphtheriae strain NCTC13129 was reported [19]. The sequence information revealed some striking features of the bacterial genome, such as the presence of as many as 13 pathogenicity islands (PAIs) [19], uncommon among C. diphtheriae strains [20]. The presence of a tox-positive prophage flanked by the att regions was confirmed and supported the findings of previous reports [21]. Despite comparable clinical importance, the genomic sequence of toxigenic $C$. ulcerans has not yet been reported. In the present study, we determined the nucleotide sequence of the toxigenic C. ulcerans isolate 0102 genome, obtained in 2001 from the pharyngeal pseudomembrane of a 52-year-old woman presenting with a sore throat and fever. This was the first toxigenic C. ulcerans infection reported in Japan. This patient had been living with nearly 20 cats before the onset of illness [22]. Details of the bacteriological characteristics of the isolate have been described elsewhere [23]. Our analysis was especially directed towards the structure of the toxpositive prophage because of its unexpectedly novel structure.

\section{Results}

\section{Genome sequence and genomic information for C. ulcerans 0102}

To determine the complete genome sequence of $C$. ulcerans 0102 , obtained short reads were assembled into five contigs by de novo assembly. Each gap was filled by direct PCR and sequencing. A circular chromosome sequence of C. ulcerans 0102 represents 2,579,188 bp, with a $\mathrm{G}+\mathrm{C}$ content of $53.4 \%$ (Additional file 1) and corresponds to the predicted restriction fragment profiles obtained by PFGE analysis (Additional file 2). The chromosome possesses 2,349 coding sequences, 51 tRNA genes, and 4 rrn rRNA operons.

\section{Comparative genome analysis of three pathogenic Corynebacterium spp}

Pair-wise sequence alignment revealed a highly conserved synteny among pathogenic Corynebacterium spp. (C. pseudotuberculosis FRC41, C. ulcerans 0102, and C. diphtheriae NCTC 13129; Figure 1). No significant genome rearrangements, such as inversion or transposition events, were observed among the three species, in accordance with previous findings [24]. The sequence similarity suggests that the chromosomes of C. ulcerans 0102 and C. pseudotuberculosis FRC41 are highly similar compared with that of $C$. diphtheriae NCTC 13129 (Figure 1). Once again, this is in accordance with previous findings in other C. ulcerans strains [24]. Similarly, a neighbor-joining phylogenetic tree, based on the partial sequence of $r p o B$, indicates that C. ulcerans 0102 is closely related with $C$. pseudotuberculosis, but clearly distinguishable from the $C$. diphtheriae clade (Additional file 3). Three prophages, ФCULC0102-I, -II, -III, were identified in C. ulcerans 0102. One of the prophages, ФCULC0102-I, carries tox, the gene encoding the diphtheria toxin (Figure 1).

\section{The tox-positive prophage of C. ulcerans 0102}

The ФCULC0102-I prophage of C. ulcerans 0102 is integrated into tRNA ${ }^{\mathrm{Arg}}$ (CULC0102_t08) (Figure 2), suggesting that the integration site is identical to that in the C. diphtheriae NCTC 13129 corynephage. In contrast, the recently reported C. ulcerans 809 and C. pseudotuberculosis FRC41 genomes possess a phage-related integrase (intC) and a nitric oxide reductase (nor) gene, respectively, instead of a prophage (Figure 2). Putative attachment sequences were similar between both prophages carrying the tox genes (Additional file 4). 


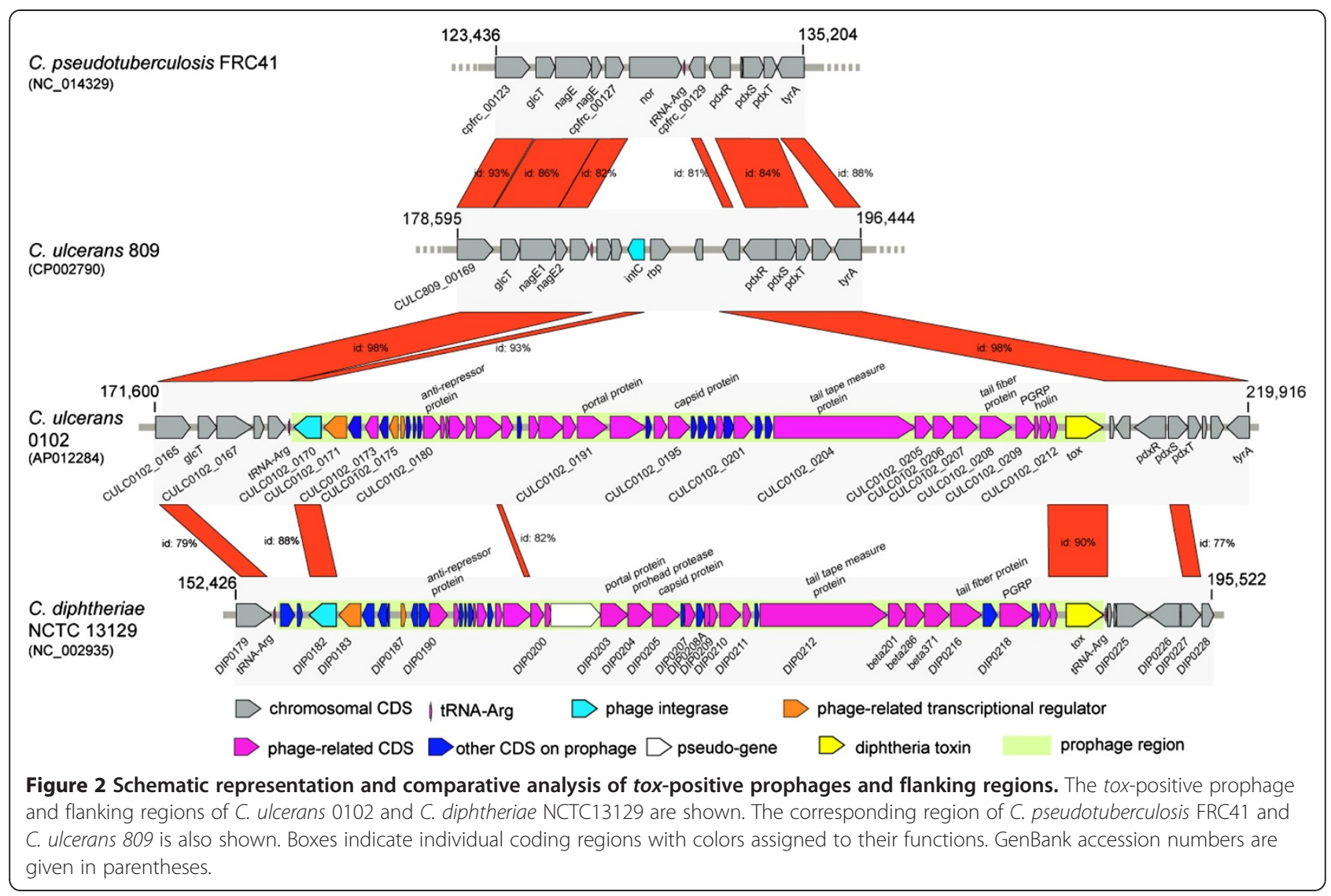

The two tox-positive prophages share the same structural features, with genes aligned in an 'integrase - packaging - head - tail - lysis - toxin' orientation (Figure 2). Pair-wise alignment of the prophages indicates a high similarity in the region encoding the putative integrase, the 3'-ends of CULC0102_0211 and CULC0102_0212, tox, and the attachment sites (Figure 2). The major phage machineries encoded in the internal phage region showed low similarity at the nucleotide and amino acid levels (less than 18\%) between C. ulcerans 0102 and $C$. diphtheriae NCTC13129.

\section{Discussion}

Whole-genome sequencing has revealed that the $C$. ulcerans 0102 genome is composed of 2,579,188 bp with a $\mathrm{G}+\mathrm{C}$ content of $53.4 \%$. These values are similar to those recently reported for C. ulcerans strains 809 $(2,502,095 \mathrm{bp}, 53.3 \% \mathrm{G}+\mathrm{C})$ and BR-AD22 (2,606,374 bp, $53.4 \% \mathrm{G}+\mathrm{C}$ ) [24]. C. ulcerans 0102 shares many common features with the two previously reported strains, including 12 virulence factors. Strain 0102 is distinctive with respect to the features of prophages integrated in its genome. It possesses a unique tox-positive prophage, ФCULC0102-I, in its chromosome (Figure 1 and Additional file 1). In the same position of the recently reported C. ulcerans 809 genome exists a remnant phage-related integrase (int $C$ ) gene [24] (Figure 2). The C. ulcerans 0102 prophage differs from the corresponding prophage in $C$. diphtheriae. Although the integrase and tox gene sequences of DCULC0102-I showed high similarity to those of the corynephage encoding tox in $C$. diphtheriae NCTC 13129, the major phage machinery genes in ФCULC0102-I are distinct from those in other corynephages in C. diphtheriae (Figure 2). This suggests that $C$. ulcerans 0102 did not immediately acquire the $C$. diphtheriae tox-positive corynephage.

There are many possible explanations for the origins of these two prophages that are tox-positive but obviously different. One of the simplest explanations we can postulate is outlined in Figure 3. Generally, bacterial prophages are duplicated by excision from chromosomal DNA and subsequent concatenation at both ends of the att sites (Figure 3A). This duplication step indicates that two highly homologous regions, int and tox, could be in close proximity and adjacent to the att site concatenation. It could be speculated that homologous recombination between two prophages may facilitate the acquisition of the tox gene in C. ulcerans 0102 from an unknown tox-positive prophage (Figure 3B) [25]. Horizontal gene transfer is one of the major mechanisms of foreign gene acquisition by bacteria, as reviewed by Ochman et al. [26]. Liu et al. have 


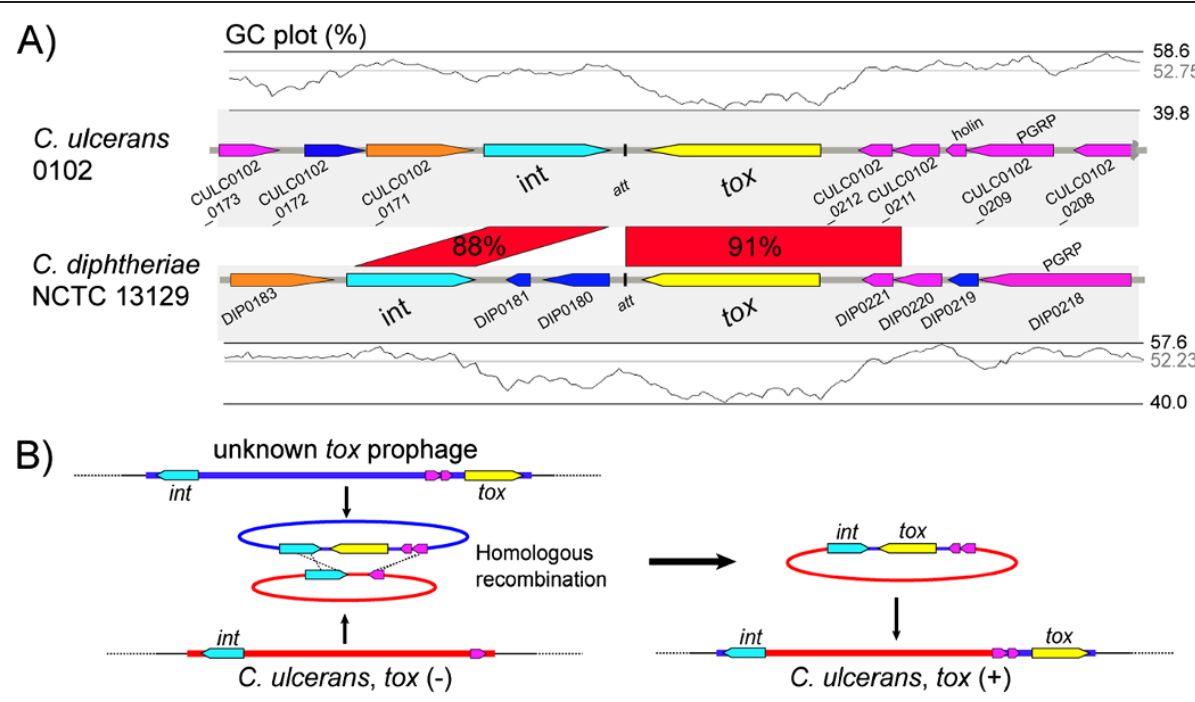

Figure 3 Schema of the diphtheria toxin acquisition hypothesis. (A) Pair-wise comparison of regions with high similarity between C. ulcerans and $C$. diphtheriae. These structures of putative phages are constructed by connecting attachment sites. The plots above and below represent the GC content calculated with a window size of 500 bp. (B) Schematic representation of how diphtheria toxin has been acquired in C. ulcerans.

demonstrated that horizontally transferred genes are often disabled and become pseudogenes. In these cases the genes are no longer beneficial to the recipients [27]. Non-toxigenic C. diphtheriae (CD450, CD119, CD448, and CD443 strains) carry tox pseudogenes that are relatively similar to the tox genes of $C$. ulcerans (Additional file 5), suggesting that horizontal gene transfer among Corynebacterium spp. might occur. Consistent with previous findings $[7,17,18,28]$, the tox gene in C. ulcerans 0102 is not identical to that of $C$. diphtheriae (Additional file 5); phylogenetic analysis of tox showed greater heterogeneity among C. ulcerans isolates than that for $C$. diphtheriae isolates (Additional file 5).

The C. diphtheriae tox gene is highly conserved among temporally and geographically diverse strains [29], therefore greater variation in tox genes from $C$. ulcerans isolates suggests that this strain might have acquired the tox gene before $C$. diphtheriae.

In a recent report, whole genome sequence analysis of non-toxigenic C. ulcerans 809 and BR-AD22 [24], the $\beta$ corynephage-like truncated integrases (CULC809_00176 and CULC22_00173) are located adjacent to the tRNA $^{\text {Arg }}$ gene, similar to ФCULC0102-I in C. ulcerans 0102 and $C$. diphtheriae. The tRNA ${ }^{\mathrm{Arg}}$ gene (CULC0102_t08) appears to be a 'hotspot' for the acquisition of $\Phi C U L C 0102-I-$ like prophages by homologous integrase.

The whole genome sequences of C. ulcerans 809 and BR-AD22 contain possible virulence factors, such as corynebacterial protease (CP40), phospholipase D (Pld), neuraminidase $(\mathrm{NanH})$, venom serine protease (Vsp1), trypsin-like serine protease (TspA), Rpf interacting protein (RpfI), cell wall-associated hydrolase $(\mathrm{CwlH})$, and five surface-anchored proteins (SpaB-F) [24]. The SpaA-type pilin, encoded by the spaABC-srtA gene cluster, is considered to play a crucial role in adhesion of $C$. diphtheriae [30]. The gene encoding the shaft protein of SpaA-type pilin (spaA) was absent in $C$. ulcerans 0102, a feature consistent with previous findings in C. ulcerans 809 and BR-AD2 [24]. As SpaB and SpaC proteins, which are assumed to be present in all three C. ulcerans strains, can contribute to host-cell adhesion in the absence of SpaA [30], this may imply a common mechanism of cell adhesion by $C$. ulcerans [24].

The C. ulcerans 809 strain was isolated from a patient with a rapid fatal pulmonary infection. The 809 strainunique virulence factor (shiga toxin-like ribosome-binding protein, $\mathrm{Rbp}$ ) is located adjacent to the truncated integrase (CULC809_00176) and corresponds to the integrase of ФCULC0102-I. It appears that virulence factors have been acquired as a cassette gene in the ФCULC0102-I-like prophage. It is intriguing to note that the 0102 strain does not carry the 809 strain-unique virulence factors ( $\mathrm{Rbp}$ and the additional venom serine protease, Vsp2), but instead carries the tox gene on ФCULC0102-I, which resulted in a diphtheria-like illness in a 52 -year-old woman.

Isolates of C. ulcerans are generally obtained from a diverse range of animals, including humans. Isolation of a human pathogen $C$. diphtheriae from animals has been reported previously, although it is rare [31]. The tox gene might be frequently transmitted through common 
prophages with the aid of the highly homologous regions among Corynebacterium spp., including C. diphtheriae and C. ulcerans isolated from animal sources.

\section{Conclusions}

Toxigenic C. ulcerans is an emerging pathogen that can be transmitted from animals to humans [5]. In the host organism, as well as in $C$. diphtheriae, the tox gene [18] is encoded by prophages. Through genome sequencing, we have identified a novel structure in a tox-positive $C$. ulcerans prophage with no significant sequence homology to those in $C$. diphtheriae. This suggests distinct origins of the prophages and thus may also explain the difference in the primary structures of their tox genes. The tox-positive bacteriophages may increase the dissemination risk of toxigenic C. ulcerans isolates, therefore, $C$. ulcerans isolates from both human and animal sources should be investigated further to determine the level of variation.

\section{Methods}

This research was not carried out on humans. No experimental research on animals was carried out.

\section{Bacterial strain}

The toxigenic $C$. ulcerans isolate 0102 was obtained in 2001 as a human clinical isolate [22,23].

\section{Preparation of genomic DNA}

Genomic DNA was isolated by conventional methods, using phenol extraction and ethanol precipitation from heat-killed bacterial cells propagated in brain-heart infusion liquid medium.

\section{Short-read DNA sequencing using an Illumina Genome Analyzer IIx}

DNA libraries of the $\sim 600$ bp insert length of $C$. ulcerans 0102 were prepared using a genomic DNA Sample Prep Kit (Illumina, San Diego, CA, USA). DNA clusters were generated on a slide using a Cluster Generation Kit (ver. 4) on an Illumina Cluster Station (Illumina), according to the manufacturer's instructions. Sequencing runs for 80-mer short reads were performed using an Illumina Genome Analyzer IIx (GA IIx) and TruSeq SBS kit v5. Fluorescent images were analyzed using the Illumina base-calling pipeline RTA2.6/SCS2.8 to obtain FASTQ-formatted sequence data.

\section{De novo assembly of short DNA reads and gap-closing}

The 80-mer reads were assembled (parameters k64, n51, c32.1373) using ABySS-pe v1.2.0 [32]. Predicted gaps were amplified with a specific PCR primer pair, followed by Sanger DNA sequencing using a BigDye Terminator v3.1 Cycle Sequencing Kit (Applied Biosystems, Foster City, CA, USA).

\section{Validation of the complete genome sequence using short-read mapping and pulsed-field gel electrophoresis (PFGE)}

To validate the genome sequence, 40-mer short reads were re-aligned with the sequence using Maq software (ver. 0.7.1) and the easyrun Perl-command [33]. Read alignment was inspected using the MapView graphical alignment viewer [34]. PFGE analysis was performed to validate the predicted restriction fragment profiles from the complete genome sequence, according to De Zoysa et al. [35]. Bacterial cells were lysed with lysozyme and protease [36], embedded in plugs, digested with the restriction endonuclease SfiI (New England Biolabs, Ipswitch, MA, USA) and electrophoresed in a CHEF DRII apparatus (Bio-Rad, Hercules, CA, USA) at $11^{\circ} \mathrm{C}$ with a pulse time of $5-20 \mathrm{~s}$ for the first $20 \mathrm{~h}$ and $1-5 \mathrm{~s}$ for the following $18 \mathrm{~h}$.

\section{Annotation and pair-wise alignment analysis}

Gene prediction from the complete sequence was performed using the NCBI Prokaryotic Genomes Automatic Annotation Pipeline (PGAAP; http://www.ncbi. nlm.nih.gov/genomes/static/pipeline.html). Several of the suggested errors were revised manually. Pseudogenes that were identified by PGAAP were checked using the read-mapping correction described above. Genomic information, such as nucleic acid variations and circular representation, was analyzed using IMC-GE software (Insilicobiology, Yokohama, Japan). A BLASTN homology search [37] was performed for the whole chromosome sequences of C. pseudotuberculosis FRC41 (accession no. NC_014329), C. ulcerans 0102, and C. diphtheriae NCTC 13129 (accession no. NC_002935). Aligned images of the homologous regions were visualized with the ACT program [38].

\section{Phylogenetic analysis}

Phylogenetic analyses of all nucleotide sequences were conducted using the neighbor-joining method with 1,000-times bootstrapping in ClustalW2 [39]. FigTree ver. 1.3.1 (http://tree.bio.ed.ac.uk/software/figtree/) software was used to display the generated tree.

\section{Nucleotide sequence accession numbers}

The complete chromosome sequence for the $C$. ulcerans 0102 strain has been deposited in the DNA Data Bank of Japan (DDBJ; accession no. AP012284). 


\section{Additional files}

Additional file: 1 Circular representation of the C. ulcerans 0102 genome. From the outside inward, the outer circle 1 indicates the size in base pairs (Mb). The red bars on Circle 2 show prophage region. Circles 3 and 4 show the positions of CDS transcribed in clockwise and anticlockwise directions, respectively. The dark blue bars on circle 5 indicate ribosomal DNA loci. Circle 6 shows a plot of $G+C$ content (in a $20 \mathrm{~kb}$ window). Circle 7 shows a plot of $\mathrm{GC}$ skew $([\mathrm{G}-\mathrm{C}] /[\mathrm{G}+\mathrm{C}]$; in a $20 \mathrm{~kb}$ window).

Additional file: 2 PFGE analysis of C. ulcerans 0102 with four restriction enzyme digestions.

Additional file: $\mathbf{3}$ Jukes-Cantor-derived phylogenetic tree based on the partial rpoB gene region among Corynebacterium isolates with 1,000-fold bootstrapping. Scale bar indicates number of substitutions per site. The number at each branch node represents the bootstrapping value. GenBank accession nos. given in parentheses.

Additional file: 4 Alignment of the nucleotide sequences of attachment site common regions among C. ulcerans 0102 and C. diphtheriae NCTC 13129. The red characters show regions annotated as tRNA ${ }^{\text {Arg }}$

Additional file: 5 Phylogenetic tree based on the tox genes among toxgenic and nontoxigenic Corynebacterium spp. using the Neighborjoining method with 1,000-fold bootstrapping. Scale bar indicates number of substitutions per site. The number at each branch node represents the bootstrapping value. GenBank accession nos. given in parentheses.

\section{Competing interests}

The authors declare that they have no competing interests.

\section{Acknowledgments}

The authors are grateful to Akio Hatanaka, Atsuhiro Tsunoda and Kenji Ooe for the 0102 clinical isolate. This work was supported by grants for Research on Emerging and Re-emerging Infectious Diseases (H23 Shinko-Ippan-007 and H22-Shinko-Ippan-010), from the Ministry of Health, Labour and Welfare, Japan.

\section{Author details}

${ }^{1}$ Laboratory of Bacterial Genomics, Pathogen Genomics Center, National Institute of Infectious Diseases, 1-23-1 Toyama, Shinjuku-ku, Tokyo 162-8640, Japan. ${ }^{2}$ Department of Bacteriology II, National Institute of Infectious Diseases, 4-7-1 Gakuen, Musashimurayama-shi, Tokyo 208-0011, Japan. ${ }^{3}$ Present address: Pharmaceutical and Medical Devices Agency, Tokyo, Japan.

\section{Authors' contributions}

TS and FT carried out the genome sequencing studies, participated in the sequence alignment and drafted the manuscript. TKo carried out maintenance, quality control and propagation of the bacterial strain for genome sequencing. AY and TKe participated in the design of the study. MT and KS conceived of and participated in coordination of the study, respectively. MK and MI coordinated the study, and drafted and finalized the manuscript. All authors read and approved the final manuscript.

Received: 10 January 2012 Accepted: 14 May 2012

Published: 14 May 2012

\section{References}

1. Bonnet JM, Begg NT: Control of diphtheria: guidance for consultants in communicable disease control. Commun Dis Public Health 1999, 2:242-249.

2. European Centre for Disease Prevention and Control: Diphtheria. In Surveillance Report: Annual epidemiological report on communicable diseases in Europe 2010. 2010:133-135.

3. Dias AASO, Silva FC, Pereira GA, Souza MC, Camello TCF, Damasceno JALD, Pacheco LGC, Miyoshi A, Azevedo VA, Hirata R, et al: Corynebacterium ulcerans isolated from an asymptomatic dog kept in an animal shelter in the metropolitan area of Rio de Janeiro, Brazil. Vector Borne Zoonotic Dis 2010, 10:743-748
4. Katsukawa C, Kawahara R, Inoue K, Ishii A, Yamagishi H, Kida K, Nishino S, Nagahama S, Komiya T, Iwaki M, Takahashi M: Toxigenic Corynebacterium ulcerans Isolated from the domestic dog for the first time in Japan. Jpn J Infect Dis 2009, 62:171-172.

5. Lartigue M-F, Monnet X, Le Flèche A, Grimont PAD, Benet J-J, Durrbach A, Fabre M, Nordmann P: Corynebacterium ulcerans in an immunocompromised patient with diphtheria and her dog. J Clin Microbiol 2005, 43:999-1001.

6. Schuhegger R, Schoerner C, Dlugaiczyk J, Lichtenfeld I, Trouillier A, Zeller-Peronnet $V$, Busch U, Berger A, Kugler R, Hörmansdorfer S, Sing A: Pigs as source for toxigenic Corynebacterium ulcerans. Emerg Infect Dis 2009, 15:1314-1315.

7. Seto Y, Komiya T, Iwaki M, Kohda T, Mukamoto M, Takahashi M, Kozaki S: Properties of corynephage attachment site and molecular epidemiology of Corynebcterium ulcerans isolated from humans and animals in Japan. Jpn J Infect Dis 2008, 61:116-122.

8. De Zoysa A, Hawkey PM, Engler K, George R, Mann G, Reilly W, Taylor D, Efstratiou A: Characterization of toxigenic Corynebacterium ulcerans strains isolated from humans and domestic cats in the United Kingdom. J Clin Microbiol 2005, 43:4377.

9. Yoshimura Y, Yamamoto A, Komiya T: A case of axillary lymph node abscess caused by percutaneous infection of Corynebacterium ulcerans through scratch by a pus-discharging cat, June 2010 (in Japanese). Infect Agents Surveillance Rep 2010, 31:331.

10. Murphy JR: Chapter 32 Corynebacterium diphtheriae. In Medical Microbiology. 4th edition. Edited by Baron S. Galveston: University of Texas Medical Branch at Galveston; 1996.

11. Pappenheimer AM Jr, Gill DM: Diphtheria. Recent studies have clarified the molecular mechanisms involved in its pathogenesis. Science 1973, 182:353-358.

12. Rappuoli R, Michel JL, Murphy JR: Integration of corynebacteriophages: tox $^{+}$, xtox $^{+}$and gtox ${ }^{+}$into two attachment sites on the Corynebacterium diphtheriae chromosome. J Bacteriol 1983, 153:1202-1210.

13. Ishii-Kanei C, Uchida T, Yoneda M: Isolation of a cured strain from Corynebacterium diphtheriae PW8. Infect Immun 1979, 25:1081-1083.

14. Cianciotto NP, Groman NB: Extended host range of a $\beta$-related corynebacteriophage. FEMS Microbiol Lett 1996, 140:221-225.

15. Oram M, Woolston JE, Jacobson AD, Holmes RK, Oram DM: Bacteriophage-based vectors for site-specific insertion of DNA in the chromosome of Corynebacteria. Gene 2007, 391:53-62.

16. Cianciotto N, Rappuoli R, Groman N: Detection of homology to the beta bacteriophage integration site in a wide variety of Corynebacterium spp. J Bacrteriol 1986, 168:103-108.

17. Sing A, Bierschenk S, Heesemann J: Classical diphtheria caused by Corynebacterium ulcerans in Germany: amino acid sequence differences between diphtheria toxins from Corynebacterium diphtheriae and $C$. ulcerans. Clin Infect Dis 2005, 40:325-326.

18. Sing A, Hogardt M, Bierschenk S, Heesemann J: Detection of differences in the nucleotide and amino acid sequences of diphtheria toxin from Corynebacterium diphtheriae and Corynebacterium ulcerans causing extrapharyngeal infections. J Clin Microbio/ 2003, 41:4848-4851.

19. Cerdeño-Tárraga A-M, Efstratiou A, Dover LG, Holden MTG, Pallen M, Bentley SD, Besra GS, Churcher C, James KD, De Zoysa A, et al: The complete genome sequence and analysis of Corynebacterium diphtheriae NCTC13129. Nucl Acids Res 2003, 31:6516-6523.

20. Iwaki M, Komiya T, Yamamoto A, Ishiwa A, Nagata N, Arakawa Y, Takahashi M: Genome organization and pathogenicity of Corynebacterium diphtheriae C7(-) and PW8 strains. Infect Immun 2010, 78:3791-3800.

21. Cianciotto N, Serwold-Davis T, Groman N, Ratti G, Rappuoli R: DNA sequence homology between attB-related sites of Corynebacterium diphtheriae, Corynebacterium ulcerans, Corynebacterium glutamicum, and the attP site of gamma-corynephage. FEMS Microbiol Lett 1990, 66:299-301.

22. Hatanaka A, Tsunoda A, Okamoto M, Ooe K, Nakamura A, Miyakoshi M, Komiya T, Takahashi M: Corynebacterium ulcerans diphtheria in Japan. Emerg Infect Dis 2003, 9:752-753.

23. Komiya T, Seto Y, De Zoysa A, Iwaki M, Hatanaka A, Tsunoda A, Arakawa Y, Kozaki S, Takahashi M: Two Japanese Corynebacterium ulcerans isolates from the same hospital: ribotype, toxigenicity and serum antitoxin titre. J Med Microbiol 2010, 59:1497-1504. 
24. Trost E, Al-Dilaimi A, Papavasiliou P, Schneider J, Viehoever P, Burkovski A, Soares SC, Almeida SS, Dorella FA, Miyoshi A, et al: Comparative analysis of two complete Corynebacterium ulcerans genomes and detection of candidate virulence factors. BMC Genomics 2011, 12:383.

25. Brüssow H, Canchaya C, Hardt W-D: Phages and the evolution of bacterial pathogens: from genomic rearrangements to lysogenic conversion. Microbiol Mol Biol Rev 2004, 68:560-602.

26. Ochman H, Lawrence JG, Groisman EA: Lateral gene transfer and the nature of bacterial innovation. Nature 2000, 405:299-304.

27. Liu Y, Harrison PM, Kunin V, Gerstein M: Comprehensive analysis of pseudogenes in prokaryotes: widespread gene decay and failure of putative horizontally transferred genes. Genome Biol 2004, 5:r64.

28. Katsukawa C, Komiya T, Yamagishi H, Ishii A, Nishino S, Nagahama S, Iwaki M, Yamamoto A, Takahashi M: Prevalence of Corynebacterium ulcerans in dogs in Osaka, Japan. J Med Microbiol 2012, 61:266-273.

29. Nakao H, Mazurova IK, Glushkevich T, Popovic T: Analysis of heterogeneity of Corynebacterium diphtheriae toxin gene, tox, and its regulatory element, $d t x R$, by direct sequencing. Res Microbiol 1997, 148:45-54.

30. Mandlik A, Swierczynski A, Das A, Ton-That H: Corynebacterium diphtheriae employs specific minor pilins to target human pharyngeal epithelial cells. Mol Microbiol 2007, 64:111-124.

31. Hall AJ, Cassiday PK, Bernard KA, Bolt F, Steigerwalt AG, Bixler D, Pawlosk LC, Whitney AM, Iwaki M, Baldwin A, et al: Novel Corynebacterium diphtheriae in domestic cats. Emerg Infect Dis 2010, 16:688-691.

32. Simpson JT, Wong K, Jackman SD, Schein JE, Jones SJM, Birol I: ABySS: a parallel assembler for short read sequence data. Genome Res 2009, 19:1117-1123.

33. Li H, Ruan J, Durbin R: Mapping short DNA sequencing reads and calling variants using mapping quality scores. Genome Res 2008, 18:1851-1858.

34. Bao H, Guo H, Wang J, Zhou R, Lu X, Shi S: MapView: visualization of short reads alignment on a desktop computer. Bioinformatics 2009, 25:1554-1555.

35. De Zoysa A, Efstratiou A, George RC, Jahkola M, Vuopio-Varkila J, Deshevoi S, Tseneva GY, Rikushin Y: Molecular epidemiology of Corynebacterium diphtheriae from northwestern Russia and surrounding countries studied by using ribotyping and pulsed-field gel electrophoresis. J Clin Microbiol 1995, 33:1080-1083.

36. Murrey BE, Singh KV, Heath JD, Sharma BR, Weinstock GM: Comparison of genomic DNAs of different enterococcal isolates using restriction endonucleases with infrequent recognition sites. J Clin Microbiol 1990, 28:2059-2063.

37. Altschul SF, Gish W, Miller W, Myers EW, Lipman DJ: Basic local alignment search tool. J Mol Biol 1990, 215:403-410.

38. Carver T, Berriman M, Tivey A, Patel C, Böhme U, Barrell BG, Parkhill Rajandream M-A: Artemis and ACT: viewing, annotating and comparing sequences stored in a relational database. Bioinformatics 2008, 24:2672-2676

39. Larkin MA, Blackshields G, Brown NP, Chenna R, McGettigan PA, McWilliam H, Valentin F, Wallace IM, Wilm A, Lopez R, et al: Clustal W and Clustal X version 2.0. Bioinformatics 2007, 23:2947-2948.

doi:10.1186/1471-2180-12-72

Cite this article as: Sekizuka et al:: Corynebacterium ulcerans 0102 carries the gene encoding diphtheria toxin on a prophage different from the C. diphtheriae NCTC 13129 prophage. BMC Microbiology 2012 12:72.

\section{Submit your next manuscript to BioMed Central and take full advantage of:}

- Convenient online submission

- Thorough peer review

- No space constraints or color figure charges

- Immediate publication on acceptance

- Inclusion in PubMed, CAS, Scopus and Google Scholar

- Research which is freely available for redistribution

Submit your manuscript at www.biomedcentral.com/submit
Biomed Central 